\title{
The Relationship Between Markers Derived From Ambulatory Blood Pressure Monitoring and Preterm Birth and Neonatal Weight in Patients With Preeclampsia
}

\section{Yifu Shi}

The Third Affiliated Hospital of Guangzhou Medical University

\section{Wenchao Cao}

The Third Affiliated Hospital of Guangzhou Medical University

\section{Zewen Feng}

The Third Affiliated Hospital of Guangzhou Medical University Haolin Mai

The Third Affiliated Hospital of Guangzhou Medical University

\section{Renjie Xie}

The Third Affiliated Hospital of Guangzhou Medical University

\section{Liuying Zhong ( $\sim 2403206927 @ q q . c o m$ )}

The Third Affiliated Hospital of Guangzhou Medical University

\section{Research Article}

Keywords: ABPM, preeclampsia, preterm birth, neonatal weight, risk prediction

Posted Date: July 6th, 2021

DOl: https://doi.org/10.21203/rs.3.rs-644267/v1

License: (c) (i) This work is licensed under a Creative Commons Attribution 4.0 International License.

Read Full License 


\section{Abstract}

Background: In order to discern the relationship between ambulatory blood pressure monitoring-related indices and preterm birth and newborn weight in patients with preeclampsia.

Methods: Sixty-seven patients with preeclampsia were included in the present study. We used logistic regression analysis to determine the relationship between blood pressure index and preterm birth risk, and to find the best threshold to predict preterm birth using an ROC curve. The relationship between the blood pressure index and neonatal weight was best described by linear regression analysis.

Results: The main result are as follows. Nocturnal diastolic blood pressure load $(\mathrm{OR}=1.045,95 \% \mathrm{Cl}$ 1.016-1.076, $\mathrm{P}=0.002$ ) has significant statistical association with preterm birth, but there was no statistical association between other indicators and preterm birth. Nocturnal systolic blood pressure, nocturnal systolic blood pressure load, and nocturnal diastolic blood pressure load each showed a significant negative correlation with neonatal weight. Other indicators were also negatively correlated, but these were not statistically significant. The predictive thresholds for nDBP load, as follows: for nDBP load at $73.8 \%$ a sensitivity of 0.78 and specificity of 0.70 .

Conclusions: In conclusion, in women with preeclampsia, elevated nDBP load has significant positive correlation with the risk of preterm birth, and nSBP, nSBP load and nDBP load has negative correlation with birth weight in newborns.

Trial registration: Not applicable

\section{Introduction}

Preeclampsia (PE) is a common obstetric and pregnancy-related disease, affecting $3-5 \%$ of all pregnancies[1]. PE exerts a significant impact on maternal and children's health[2], and it is also a significant cause of maternal mortality and morbidity[3]. Blood pressure levels during pregnancy in patients with preeclampsia-especially in the hypertensive range-have long been considered to be risk factors for pregnancy complications and poor perinatal outcomes[4][5][6]. Evaluation of blood pressure is usually based on traditional measurement methods, but there are some problems in assessing blood pressure during pregnancy. For example, physician preference for the ultimate iteration of blood pressure readings will significantly impact the measurement[7]. Ambulatory blood pressure monitoring (ABPM) has been shown to outperform traditional blood pressure screening in clinical work for predicting targetorgan damage with hypertension, and cardiovascular events and drug efficacy[8]. ABPM can also be used to diagnose white-coat hypertension and hidden hypertension that traditional measurement cannot achieve[9][10]. However, the most significant difference between ABPM and traditional measurements is that ABPM can be measured continuously without time or place restrictions (especially at night), while traditional measurements are typically delimited by location and time, and dynamic changes in blood pressure cannot be obtained. Many scholars have described research foci in this area. Some investigators[11] have used ABPM to predict preeclampsia, and some new ABPM-based indicators have 
also been proposed and confirmed to be helpful in identifying gestational hypertension-including a diurnal variation in blood pressure[12] and a hypertension index[13]. Preterm birth is one of the important adverse outcomes of preeclampsia, and one study[14] revealed that measuring prenatal blood pressure can predict preterm birth in preeclamptic patients. Contemporaneous studies[15] showed that 24-hour blood pressure monitoring was more predictive of preterm birth than traditional measurements in patients with gestational hypertension.

Moreover, the relationship between ABPM components and a newborn's birth weight as another important pregnancy-related outcome has not yet been determined. It was reported that maternal mean diurnal diastolic blood pressure (DBP) levels were negatively correlated with birth weight[16][17]. However, few authors have reported a prediction of birth weight in eclamptic patients.

With this study, we therefore aimed to explore the role of ABPM in predicting preterm birth in patients with preeclampsia.

\section{Methods}

Overall design

This retrospective case analysis included patients in antenatal care at the Third Affiliated Hospital of Guangzhou Medical University from January 2014 to October 2019. We examined the respective electronic medical records and medical supervision records to determine patients to be included for final analysis, screening for both inclusion and exclusion criteria. We ultimately assessed the relationship between ABPM-derived indicators and the risk of preterm birth and neonatal weight.

Population

Our inclusion criteria were (1) the availability of ABPM during the study period; and (2) a discharge diagnosis of any type of pre-eclampsia, including preeclampsia and severe preeclampsia.

The exclusion criteria were (1) a diagnosis of chronic medical diseases (e.g., chronic hypertension, heart disease, diabetes, and hepatitis), intrahepatic cholestasis of gestation, or gestational diabetes mellitus; (2) confirmed multiple pregnancies; (3) invalid ABPM data; and (4) the absence of important ABPMderived indicators or outcomes.

Population characters

Maternal age, BMI, and gestational age at the time of delivery, and obstetric history were retrieved from the electronic medical records.

ABPM-derived indicator data

ABPM-derived indicator data were collected by dynamic blood pressure-monitoring devices(TM2430; A\&D, Tokyo), which have already been proven to be well-tolerated and shown to be of value for pregnant 
women[18]. ABPM was performed within 1 week after admission to understand the changes in blood pressure. This dynamic blood pressure-monitoring device regularly measures a wearer's BP every 20 minutes between 06:00 and 21:59 h, and every 30 minutes between 22:00 and 05:59 h. Only ABPM cycles with a qualified measurement rate of more than $90 \%$ were included in the final analysis.

To further uncover the clinical value of ABPM, we not only entered the conventional mean diurnal systolic blood pressure (dSBP), mean diurnal diastolic blood pressure (dDBP), mean nocturnal diastolic blood pressure (nDBP), and mean nocturnal systolic blood pressure (nSBP), but we also entered the diurnal systolic blood pressure load (dSBP load), diurnal diastolic blood pressure load (dDBP load), nocturnal systolic blood pressure load (nSBP load), and nocturnal diastolic blood pressure load (nDBP load). The load value of blood pressure was calculated by comparing the reading with the established hypertension threshold (Diurnal: $135 \mathrm{~mm} \mathrm{Hg}$ for systolic blood pressure and $85 \mathrm{~mm} \mathrm{Hg}$ for diastolic blood pressure. Nocturnal: $120 \mathrm{~mm} \mathrm{Hg}$ for systolic blood pressure and $70 \mathrm{~mm} \mathrm{Hg}$ for diastolic blood pressure.), and then calculating the percentage of the valid dynamic blood pressure measurement that was above the established threshold.

\section{Obstetric information}

PE was defined as the presence of systolic blood pressure $\geq 140 \mathrm{~mm} \mathrm{Hg}$ and/or diastolic blood pressure $\geq 90 \mathrm{~mm} \mathrm{Hg}$; accompanied by urinary protein $\geq 0.3 \mathrm{~g} / 24 \mathrm{~h}$, or random urinary protein $\geq(+)$, or no proteinuria after 20 weeks of gestation; and combined with any of the following: thrombocytopenia (platelets $<100 \times 10^{9} / \mathrm{L}$ ), liver function impairment (serum transaminase level greater than twice normal), renal function impairment (serum creatinine level above $1.1 \mathrm{mg} / \mathrm{dl}$ or more than twice normal); or pulmonary edema or new central nervous system or visual impairment[1]. Premature delivery was defined as the birth of a baby at a gestational age of less than 37 weeks and with low birth weight $(<2500 \mathrm{~g})[9]$.

Statistical analysis

We collected and cleansed data using MS Excel software, and performed statistical analysis with SPSS 25.0 software (SPSS Co. Ltd., Shanghai, China). Numerical data that followed a normal distribution are presented as mean \pm standard deviation $(x \pm S D$ ), and numerical data with a skewed distribution are presented as medians (M [P25, P75]). Means were compared using a $t$ test for normally distributed continuous variables, and the non-parametric rank-sum test for continuous variables showing a skewed distribution. To study the relationship between blood pressure and premature delivery, we first do a singlefactor logistics regression analysis to select indicators with small $p$ values $(p<0.1)$ and incorporate them into a multi-factor regression model, then we used multi-factor logistics regression(forward progressive) and calculated the odds ratio (OR) and its $95 \%$ confidence interval (Cl). The relationship between blood pressures and birth weight was assessed using linear regression, and receiver operating characteristic (ROC) curves were used for the prediction of premature delivery and the evaluation of the optimal threshold (Fig. 1). A p value of $\leq 0.05$ was considered statistically significant. 


\section{Results}

Sixty-seven pregnant women with a discharge diagnosis of any kind of pre-eclampsia were ultimately included in the analysis. There were three women with fetal death, and these data were not included in any subsequent analyses. A total of 37 women underwent preterm birth, while 27 did not. Neither age $(33[28,37]$ vs. $30[28,34], P=0.27)$ nor $B M I(23.00[20.84,25.37]$ vs. $23.00[20.40,25.22], P=0.854)$ was significantly different between the two groups. Those women who gave birth prematurely exhibited a significantly lower neonatal birth weight than those who delivered at the appropriate time $(1745.73 \pm$ 621.80 vs. $3017.37 \pm 538.11, \mathrm{P}<0.001)$. The $\operatorname{nDBP}(82.86 \pm 8.89$ vs. $77.44 \pm 9.09, \mathrm{P}=0.023)$ and $\mathrm{nDBP}$ load $(94.44[73.90,100.00]$ vs. $62.90[46.67,77.78], P<0.001)$ for the preterm group were significantly higher than those of the non-preterm group, but the other indices did not demonstrate significant differences. Detailed information of the women included in our study is listed in Table 1. 
Comparison of basic clinical data between the two groups. Numerical data following a normal distribution are presented as mean \pm standard deviation $(\mathrm{x} \pm \mathrm{SD})$, and numerical data following a skewed distribution are presented as medians (M [P25, P75]).

\begin{tabular}{|llll|}
\hline & Premature delivery & Non-premature delivery & P value \\
& N=37 & N=27 & \\
\hline Age (years) & $33(28,37)$ & $30(28,34)$ & 0.270 \\
\hline BMI (kg/m $\left.{ }^{2}\right)$ & $23.00(20.84,25.37)$ & $23.00(20.40,25.22)$ & 0.854 \\
\hline Gestational age (weeks) & $34.00(31.00,35.50)$ & $38.00(37.00,39.00)$ & $<0.001$ \\
\hline Number of pregnancies & $2.00(1.00,3.00)$ & $1.00(1.00,2.00)$ & 0.027 \\
\hline Neonatal birth weight (g) & $1745.73 \pm 621.80$ & $3017.37 \pm 538.11$ & $<0.001$ \\
\hline dSBP (mm Hg) & $135.51 \pm 12.41$ & $134.81 \pm 13.56$ & 0.834 \\
\hline dSBP load (\%) & $44.40(21.60,80.90)$ & $43.90(17.4,77.78)$ & 0.833 \\
\hline dDBP (mm Hg) & $86.54 \pm 8.89$ & $84.37 \pm 8.18$ & 0.323 \\
\hline $\begin{array}{l}\text { dDBP load (\%) } \\
\text { nSBP (mm Hg) }\end{array}$ & $54.06 \pm 30.85$ & $47.60 \pm 31.12$ & 0.413 \\
\hline nSBP load (\%) & $130.69 \pm 11.05$ & $129.82 \pm 16.04$ & 0.80 \\
\hline nDBP (mm Hg) & $67.02(52.78,91.67)$ & $67.02(44.44,87.5)$ & 0.444 \\
\hline nDBP load (\%) & $82.86 \pm 8.89$ & $77.44 \pm 9.09$ & 0.023 \\
\hline $\begin{array}{l}\text { dSBP: diurnal systolic blood pressure, dDBP: diurnal diastolic blood pressure, nDBP: nocturnal } \\
\text { diastolic blood pressure, nSBP: nocturnal systolic blood pressure, dSBP load: diurnal systolic blood } \\
\text { pressure load, dDBP load: diurnal diastolic blood pressure load, nSBP load: nocturnal systolic blood } \\
\text { pressure load, nDBP load: nocturnal diastolic blood pressure load. A p value of } \leq 0.05 \text { was considered } \\
\text { statistically significant. }\end{array}$ & & & $<0.001$ \\
\hline
\end{tabular}

Preterm birth and neonatal birth weight were the two primary outcomes of our study. In the single-factor logistics regression analysis, only nDBP, nDBP load and the number of pregnancies has $\mathrm{p}$ values of less than 0.1 (Table 2). In the models eventually built through multi-factor logistics regression (Table 3), nDBP is removed from the model in progressive forward analysis, the effect of nDBP load $(\mathrm{OR}=1.045,95 \% \mathrm{Cl}$ $1.016-1.076, P=0.002)$ and the number of pregnancies $(\mathrm{OR}=1.799,95 \% \mathrm{Cl} 1.031-3.139, \mathrm{P}=0.039)$ were statistically significant on the risk of preterm birth (Table 4). 
Table 2

Single-factor regression analysis results

\begin{tabular}{|lllllll|}
\hline indexes & b value & SE & Wald & OR & $95 \%$ Cl & P value \\
\hline Age & 0.048 & 0.052 & 0.934 & 1.049 & $0.947-1.162$ & 0.361 \\
\hline BMI & 0.058 & 0.075 & 0.603 & 1.060 & $0.915-1.228$ & 0.438 \\
\hline Number of pregnancies & 0.459 & 0.235 & 3.801 & 1.582 & $0.998-2.508$ & 0.051 \\
\hline dSBP & 0.004 & 0.020 & 0.047 & 1.004 & $0.966-1.044$ & 0.828 \\
\hline dSBP load & 0.002 & 0.008 & 0.039 & 1.002 & $0.986-1.018$ & 0.843 \\
\hline dDBP & 0.030 & 0.030 & 0.997 & 1.031 & $0.971-1.094$ & 0.318 \\
\hline dDBP load & 0.007 & 0.008 & 0.689 & 1.007 & $0.991-1.024$ & 0.406 \\
\hline nSBP & 0.005 & 0.019 & 0.067 & 1.005 & $0.968-1.043$ & 0.796 \\
\hline nSBP load & 0.011 & 0.009 & 1.418 & 1.011 & $0.993-1.029$ & 0.234 \\
\hline nDBP & 0.070 & 0.032 & 4.934 & 1.073 & $1.008-1.142$ & 0.026 \\
\hline nDBP load & 0.040 & 0.013 & 9.293 & 1.041 & $1.014-1.068$ & 0.002 \\
\hline
\end{tabular}

Table 3

Multi-factor logistics regression(forward progressive) analysis results

\begin{tabular}{|lllllll|}
\hline indexes & b value & SE & Wald & OR & $\mathbf{9 5 \%}$ Cl & P value \\
\hline Number of pregnancies & 0.587 & 0.284 & 4.276 & 1.799 & $1.031-3.139$ & 0.039 \\
\hline nDBP load & 0.044 & 0.015 & 9.274 & 1.045 & $1.016-1.076$ & 0.002 \\
\hline
\end{tabular}


Table 4

The relationships among blood pressure, gestational age, and neonatal birth weight $(\mathrm{g})$.

\begin{tabular}{|c|c|c|c|}
\hline Blood pressure indices & $\begin{array}{l}\text { Neonatal birth weight }(\mathrm{g}) \text { - } \\
\text { beta } \pm \mathrm{SE} \\
\text { per } 1 \mathrm{~mm} \mathrm{Hg}(1 \%) \\
\text { change in BP (BP load) }\end{array}$ & $\begin{array}{l}\text { Neonatal birth weight }(\mathrm{g}) \text { - } \\
\text { beta } \pm \mathrm{SE} \\
\text { per weeks } \\
\text { change in gestational age }\end{array}$ & Adjusted $\mathrm{R}^{2}$ \\
\hline dSBP & $-6.117 \pm 4.762$ & $199.137 \pm 17.234^{\star \star}$ & 0.691 \\
\hline dSBP load & $-1.944 \pm 1.917$ & $201.058 \pm 17.19^{\star \star}$ & 0.687 \\
\hline dDBP & $-4.692 \pm 7.241$ & $200.061 \pm 17.596^{\star \star}$ & 0.684 \\
\hline dDBP load & $-1.042 \pm 2.011$ & $200.694 \pm 17.555^{\star \star}$ & 0.683 \\
\hline $\mathrm{nSBP}$ & $-9.165 \pm 4.389^{*}$ & $204.618 \pm 16.732^{\star *}$ & 0.703 \\
\hline nSBP load & $-6.818 \pm 1.973^{\star \star}$ & $202.74 \pm 15.806^{\star *}$ & 0.734 \\
\hline $\mathrm{nDBP}$ & $-9.583 \pm 6.624$ & $196.88 \pm 17.417^{\star \star}$ & 0.693 \\
\hline nDBP load & $-5.654 \pm 2.328^{*}$ & $187.247 \pm 17.644^{\star \star}$ & 0.710 \\
\hline
\end{tabular}

In the linear regression model (Table 4), we added gestational age as an independent variable to the regression model, and ABPM blood pressure components and gestational weeks were used to predict neonatal weight. We observed a negative correlation between all indicators and neonatal weight. However, only nSBP $(b=-9.165 \pm 4.389 ; P=0.041)$, nSBP load $(b=-6.818 \pm 1.973 ; P=0.001)$, and nDBP load $(b=-5.654 \pm 2.328 ; P=0.018)$ were statistically correlated. We also noted a significant positive correlation between the number of gestational weeks and neonatal weight $(P<0.001)$. The adjusted $R^{2}$ for all models was greater than 0.68 (range, $0.68-0.73$ ), with the greatest impact on neonatal weight being nSBP.

To better explore ABPM-derived indicator values in predicting the occurrence of preterm birth and to discern the appropriate cut-off point for ABPM, we constructed ROC curves (Fig. 1) to describe the relationship between different ABPM-derived indicators and preterm birth. Among the blood pressure components, nDBP load possessed predictive value for the measurement of preterm labor, We found predictive thresholds for nDBP load, as follows: for nDBP load at $73.8 \%$ a sensitivity of 0.78 and specificity of 0.70 .

\section{Discussion}


With the wide application of ABPM overall, the application of ABPM in pregnant women specifically is also increasing. Some investigators[9][19][20] have proposed the use of ABPM to diagnose gestational hypertension and differentiate it from white-coat hypertension, while others applied ABPM to patients with gestational hypertension and predicted small-for-gestational-age fetuses[21]. As one of the common diseases of pregnant women, preeclampsia has been studied extensively. In the Martin study[11], the nocturnal blood pressure of patients with high-risk mid-term pregnancy was measured to predict the occurrence of pre-eclampsia, and these authors analyzed the relationship between circadian bloodpressure rhythm and adverse outcomes in patients with eclampsia[22]. However, we did not find any studies in which various BPs were applied to the prediction of preterm birth and neonatal weight in preeclampsia, and that is why we chose to study this further.

We founded the relationship between ABPM and the risk of preterm birth in preeclampsia during this retrospective study. In general, the higher the blood pressure levels, the greater the risk of preterm birth; however, only nDBP load were statistically significant. We next uncovered the threshold point with the greatest value in predicting preterm birth risk through the ROC curve (nDBP load at 73.8\% showed a sensitivity of 0.78 and specificity of 0.70 ). According to ABPM and gestational weeks, we herein established a predictive model of neonatal weight in patients with preeclampsia. Blood pressure level and neonatal weight were negatively correlated, while gestational weeks were positively correlated with neonatal weight. All models showed good fit and availability $\left(R^{2}>0.68\right)$.

Interpretation of results

In patients with preeclampsia, blood pressure was generally high-most likely due to systemic arteriolar spasms, endothelial injury, and local ischemia[23]. The principal mechanism of nocturnal blood pressure elevation involves fluid volume retention and/or activation of the sympathetic nervous system[24], and it was reported that women with preeclampsia exhibited impaired baroreflex sensitivity[25], which may be one of the causes of the increased nocturnal blood pressure. Sleep-pattern disruption in preeclamptic patients may, however, also be a cause of nocturnal blood pressure increase[26].

Of the 67 subjects included in our study, 37 women underwent preterm births, and elevated nDBP load was positively associated with the risk of preterm birth. Lior et al.[15] showed that 24-hour elevated blood pressure increased the risk of preterm birth, but the difference between studies was that ours was conducted in women with preeclampsia, while theirs was in pregnant and hypertensive women. The aforementioned authors used a 24-hour mean blood pressure, but in order to fully explore the value of ABPM, we distinguished between diurnal and nocturnal blood pressure, and also provided a groundbreaking analysis of the relationship between blood pressure load and these outcomes. In our analysis, nDBP load manifested a better predictive value and stronger statistical significance, and we speculate that this may be because the duration of the nocturnal blood pressure rise was too protracted-leading to more severe placental dysfunction, and further leading to developmental injury to the fetus. In addition, 
we constructed a ROC curve based on the relationship between nDBP load and preterm birth, and obtained an optimal threshold (for nDBP load at $73.8 \%$, a sensitivity of 0.78 and specificity of 0.70 ). In our investigation, nSBP, nSBP load, and nDBP were negatively correlated with neonatal weight, and some studies[18] have shown similar results, although these authors used clinical blood pressure instead of ABPM. In the study by Waugh [16], there was a significant negative correlation between diurnal dynamic diastolic blood pressure measurements and birth weight (gradient, $-13.5 ; 95 \% \mathrm{Cl},-25.4$ to -3.0 ), and other ambulatory blood pressure measurements and birth weight were also negatively correlated.

It has been reported[27] that preeclampsia increases the risk of preterm birth, and some studies[28][29] have indicated that the core of preeclampsia is placental damage. In preeclampsia, the incidence of placental blood flow interruption and the risk of ischemia/reperfusion injury are increased, and intermittent blood flow can alter placental development, with placental dysfunction leading to impaired fetal development. These investigators also found that low-birth-weight infants were more prone to neonatal infection, perinatal respiratory distress, jaundice, hypoglycemia, erythrocytosis, hypothermia, and other adverse outcomes[30]. A large number of epidemiologic studies also showed that low birth weight in infants was closely related to adult diseases such as type 2 diabetes, obesity, metabolic syndrome, hypertension, chronic cardiovascular disease, and chronic lung disease, and that it can be inherited by the subsequent generation[31][32].

Our results are of positive clinical significance and can provide a reference to avoid preterm birth and low birth weight by controlling blood pressure-thus avoiding a series of adverse secondary outcomes. Furthermore, because reference values for dynamic systolic/diastolic blood pressures during pregnancy have not been determined, clinicians cannot judge whether women are at risk with respect to ABPM during pregnancy. Our study therefore provides a feasible reference value for the clinical establishment of risk for premature delivery in preeclamptic patients.

\section{Limitations}

This study has several limitations. First, compared with other studies, our sample size was relatively small. Second, a report[19] showed that the early termination of pregnancy can reduce the incidence of adverse outcomes in patients with preeclampsia, and in our data collection we did not consider which women ended their pregnancies early to avoid adverse outcomes. This omission may thus create a discrepancy in our study results. Finally, the model we used lacked external validation, and was therefore only a theoretical study that requires further research to confirm its clinical predictive value. In addition, the conclusion of this study cannot be generalized to all pregnant women, and this is a shortcoming of our research.

\section{Conclusion}

In conclusion, in women with preeclampsia, elevated nDBP load has significant positive correlation with the risk of preterm birth, and nSBP, nSBP load and nDBP load has negative correlation with birth weight in newborns. 


\section{Declarations}

\section{Ethics approval and consent to participate}

This study has been approved by the Medical Ethics Committee of The Third Affiliated Hospital of Guangzhou Medical University.

\section{Consent for publication}

Not applicable

\section{Availability of data and materials}

The datasets during the current study available from the corresponding author on reasonable request.

\section{Competing interests}

No potential conflict of interest was reported by the authors.

\section{Funding}

Not applicable.

\section{Author Contributions}

Concept and design: Yifu Shi, Wenchao Cao, Zewen Feng, Haolin Mai, Renjie Xie

Acquisition, analysis, or interpretation of data: Yifu Shi, Wenchao Cao, Zewen Feng, Haolin Mai

Drafting of the manuscript: Yifu Shi, Wenchao Cao, Zewen Feng, Haolin Mai

Critical revision of the manuscript for important intellectual content: Yifu Shi, Renjie Xie, Liuying Zhong

Statistical analysis: Yifu Shi, Wenchao Cao

Supervision: Renjie Xie, Liuying Zhong

All authors have read and approved the manuscript.

\section{Acknowledgements}

We thank LetPub (www.letpub.com) for its linguistic assistance during the preparation of this manuscript.

\section{References}


1. ACOG Practice Bulletin No. 202: Gestational Hypertension and Preeclampsia. Obstetrics and gynecology 2019, 133(1):1.

2. Chen CW, Jaffe IZ, Karumanchi SA: Preeclampsia and cardiovascular disease. Cardiovasc Res 2014, 101(4):579-586.

3. Saleem S, McClure E, Goudar S, Patel A, Esamai F, Garces A, Chomba E, Althabe F, Moore J, Kodkany $B$ et al: A prospective study of maternal, fetal and neonatal deaths in low- and middle-income countries. Bulletin of the World Health Organization 2014, 92(8):605-612.

4. Hnat M, Sibai B, Caritis S, Hauth J, Lindheimer M, MacPherson C, VanDorsten J, Landon M, Miodovnik M, Paul $\mathrm{R}$ et al: Perinatal outcome in women with recurrent preeclampsia compared with women who develop preeclampsia as nulliparas. American journal of obstetrics and gynecology 2002, 186(3):422-426.

5. Abramovici D, Friedman S, Mercer B, Audibert F, Kao L, Sibai B: Neonatal outcome in severe preeclampsia at 24 to 36 weeks' gestation: does the HELLP (hemolysis, elevated liver enzymes, and low platelet count) syndrome matter? American journal of obstetrics and gynecology 1999, 180:221225.

6. Gofton E, Capewell V, Natale R, Gratton R: Obstetrical intervention rates and maternal and neonatal outcomes of women with gestational hypertension. American journal of obstetrics and gynecology 2001, 185(4):798-803.

7. Villar J, Repke J, Markush L, Calvert W, Rhoads G: The measuring of blood pressure during pregnancy. American Journal of Obstetrics and Gynecology 1989, 161(4):1019-1024.

8. Pickering T, Hall J, Appel L, Falkner B, Graves J, Hill M, Jones D, Kurtz T, Sheps S, Roccella E: Recommendations for blood pressure measurement in humans and experimental animals: part 1: blood pressure measurement in humans: a statement for professionals from the Subcommittee of Professional and Public Education of the American Heart Association Council on High Blood Pressure Research. Circulation 2005, 111(5):697-716.

9. Brown M, Magee L, Kenny L, Karumanchi S, McCarthy F, Saito S, Hall D, Warren C, Adoyi G, Ishaku S: Hypertensive Disorders of Pregnancy: ISSHP Classification, Diagnosis, and Management Recommendations for International Practice. Hypertension (Dallas, Tex : 1979) 2018, 72(1):24-43.

10. Mosimann B, Pfiffner C, Amylidi-Mohr S, Risch L, Surbek D, Raio L: First trimester combined screening for preeclampsia and small for gestational age - a single centre experience and validation of the FMF screening algorithm. Swiss medical weekly 2017, 147:w14498.

11. Salazar M, Espeche W, Leiva Sisnieguez C, Leiva Sisnieguez B, Balbín E, Stavile R, March C, Olano R, Soria $\mathrm{A}$, Yoma $\mathrm{O}$ et al: Nocturnal hypertension in high-risk mid-pregnancies predict the development of preeclampsia/eclampsia. Journal of hypertension 2019, 37(1):182-186.

12. Ayala D, Hermida R, Mojón A, Fernández J, Silva I, Ucieda R, Iglesias M: Blood pressure variability during gestation in healthy and complicated pregnancies. Hypertension (Dallas, Tex : 1979) 1997, 30:611-618. 
13. Hermida R, Ayala D, Mojón A, Fernández J, Silva I, Ucieda R, Iglesias M: Blood pressure excess for the early identification of gestational hypertension and preeclampsia. Hypertension (Dallas, Tex : 1979) 1998, 31(1):83-89.

14. Macdonald-Wallis C, Silverwood R, de Stavola B, Inskip H, Cooper C, Godfrey K, Crozier S, Fraser A, Nelson S, Lawlor D et al: Antenatal blood pressure for prediction of preeclampsia, preterm birth, and small for gestational age babies: development and validation in two general population cohorts. BMJ (Clinical research ed) 2015, 351:h5948.

15. Liro M, Gasowski J, Wydra D, Grodzicki T, Emerich J, Narkiewicz K: Twenty-four-hour and conventional blood pressure components and risk of preterm delivery or neonatal complications in gestational hypertension. Blood pressure 2009, 18:36-43.

16. Waugh J, Perry I, Halligan A, De Swiet M, Lambert P, Penny J, Taylor D, Jones D, Shennan A: Birth weight and 24-hour ambulatory blood pressure in nonproteinuric hypertensive pregnancy. American journal of obstetrics and gynecology 2000, 183(3):633-637.

17. Steer P, Little M, Kold-Jensen T, Chapple J, Elliott P: Maternal blood pressure in pregnancy, birth weight, and perinatal mortality in first births: prospective study. BMJ (Clinical research ed) 2004, 329(7478):1312.

18. Palatini P, Frigo G, Bertolo $O$, Roman E, Da Cortà R, Winnicki M: Validation of the A\&D TM-2430 device for ambulatory blood pressure monitoring and evaluation of performance according to subjects' characteristics. Blood pressure monitoring 1998, 3(4):255-260.

19. Regitz-Zagrosek V, Roos-Hesselink J, Bauersachs J, Blomstrom-Lundqvist C, Cifkova R, De Bonis M, lung B, Johnson M, Kintscher U, Kranke P et al: 2018 ESC Guidelines for the management of cardiovascular diseases during pregnancy. Kardiologia polska 2019, 77(3):245-326.

20. Brown M: Is there a role for ambulatory blood pressure monitoring in pregnancy? Clinical and experimental pharmacology \& physiology 2014, 41(1):16-21.

21. Eguchi K, Ohmaru T, Ohkuchi A, Hirashima C, Takahashi K, Suzuki H, Kario K, Matsubara S, Suzuki M: Ambulatory BP monitoring and clinic BP in predicting small-for-gestational-age infants during pregnancy. Journal of human hypertension 2016, 30(1):62-67.

22. Zhong L, Deng W, Zheng W, Yu S, Huang X, Wen Y, Chiu P, Lee C: The relationship between circadian blood pressure variability and maternal/perinatal outcomes in women with preeclampsia with severe features. Hypertension in pregnancy 2020, 39(4):405-410.

23. Mol B, Roberts C, Thangaratinam S, Magee L, de Groot C, Hofmeyr G: Preeclampsia. Lancet (London, England) 2016, 387(10022):999-1011.

24. Kario K: Proposal of a new strategy for ambulatory blood pressure profile-based management of resistant hypertension in the era of renal denervation. Hypertension research : official journal of the Japanese Society of Hypertension 2013, 36(6):478-484.

25. Silver $\mathrm{H}$, Tahvanainen $\mathrm{K}$, Kuusela T, Eckberg D: Comparison of vagal baroreflex function in nonpregnant women and in women with normal pregnancy, preeclampsia, or gestational hypertension. American journal of obstetrics and gynecology 2001, 184(6):1189-1195. 
26. Ekholm E, Polo O, Rauhala E, Ekblad U: Sleep quality in preeclampsia. American journal of obstetrics and gynecology 1992, 167(5):1262-1266.

27. Tannetta D, Sargent I: Placental disease and the maternal syndrome of preeclampsia: missing links? Current hypertension reports 2013, 15(6):590-599.

28. Jim B, Karumanchi S: Preeclampsia: Pathogenesis, Prevention, and Long-Term Complications. Seminars in nephrology 2017, 37(4):386-397.

29. Bakker R, Steegers $E$, Hofman A, Jaddoe V: Blood pressure in different gestational trimesters, fetal growth, and the risk of adverse birth outcomes: the generation $\mathbf{R}$ study. American journal of epidemiology 2011, 174(7):797-806.

30. Lv Ying, Shao Jie: Birth recognition and research Progress. Chinese Journal of Practical Pediatrics 2019, 34(10): 825-829.

31. Lal M, Manktelow B, Draper E, Field D: Chronic lung disease of prematurity and intrauterine growth retardation: a population-based study. Pediatrics 2003, 111(3):483-487.

32. Thamotharan M, Garg M, Oak S, Rogers L, Pan G, Sangiorgi F, Lee P, Devaskar S: Transgenerational inheritance of the insulin-resistant phenotype in embryo-transferred intrauterine growth-restricted adult female rat offspring. American journal of physiology Endocrinology and metabolism 2007, 292(5):E1270-1279.

\section{Figures}

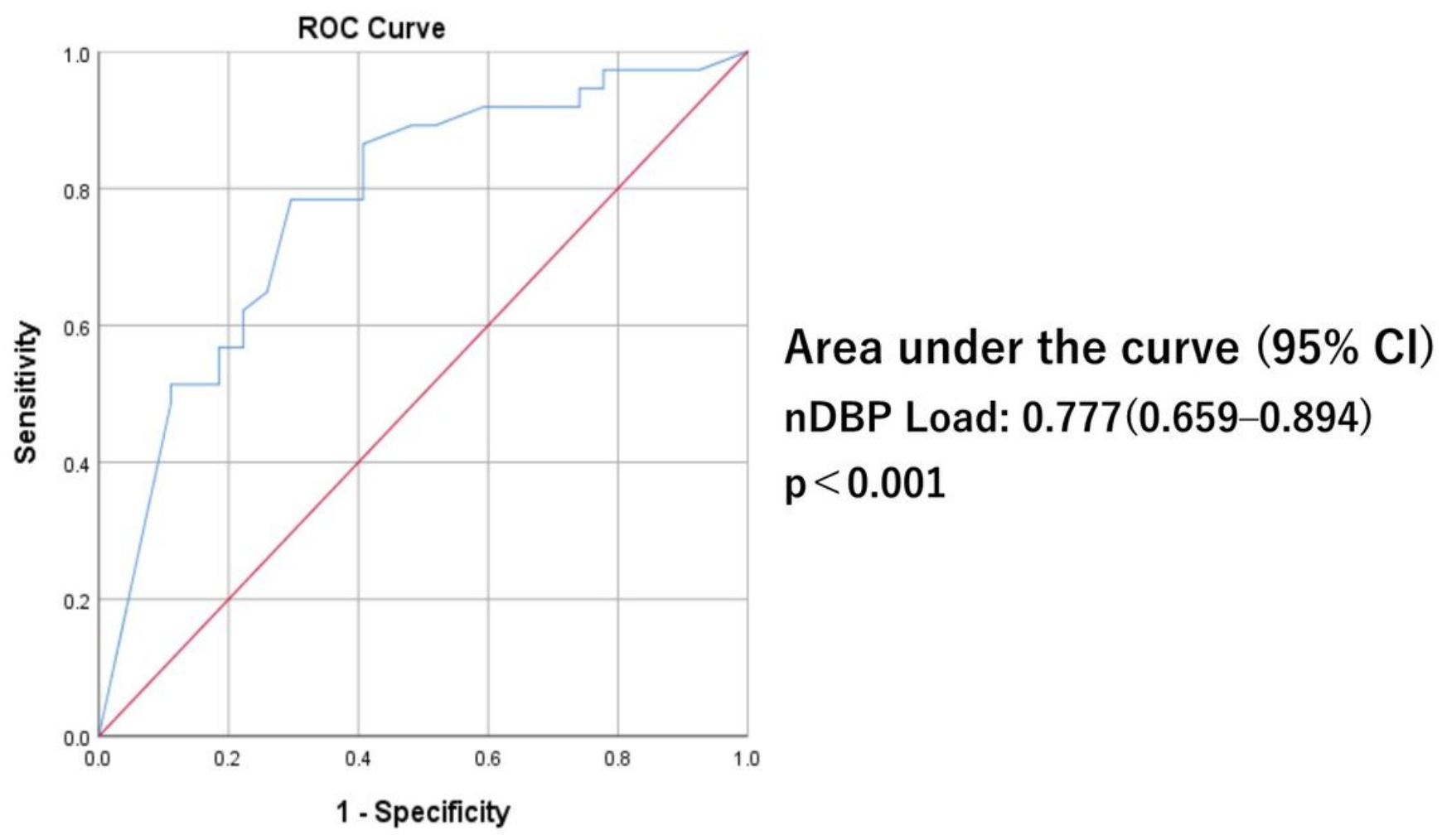

Diagonal segments are produced by ties. 


\section{Figure 1}

ROC curves for $A B P M$ in the prediction of premature delivery. The areas under the curve was 0.777 for nDBP load. The predictive thresholds for nDBP load, as follows: for $\mathrm{nDBP}$ load at $73.8 \%$ a sensitivity of 0.78 and specificity of 0.70 . 